\title{
Sensors for Smart Systems - Requirements to Sensor Systems
}

Heinze, Lars

VDI/VDE Innovation + Technik GmbH

Steinplatz 1, 10623 Berlin, Germany, T +49 30310078 165, E heinze@vdivde-it.de

\section{Smart Systems}

Smart Systems in the sense of the Strategic Research Agenda (SRA) defined by the European Technology Platform on Smart Systems Integration (EPoSS / www.smart-systems-integration.org) are self-diagnosis and possess sensoric and actoric capabilities. This compination of features enables them to describe and to evaluate situations. They might be embedded in other products, because of their miniuaturisation. They can decide and communicate with their environment including other Smart Systems. Therefore these systems will need cognitive skills with at least certain intelligence. As a result of such intelligence 'smart' behaviour is expected.

Smart Systems with such cognitve qualities will be technical systems possessing perception and awareness. They are miniaturised, highly reliable, often networked (wireless), autonomous and implantable. Overall these are qualities like self-organisation, adaptation, individualisation and personalisation as well as certain autonomy. The implementation of such qualities would enable the evolution of classical Microsystems towards Smart Systems. Figure 1 displays a possible path of transition from Microsystems to a Smart Systems.

In conventional Microsystems, actions are based on a sensor(-element) signal which was processed by a Signal Processing Unit based on an implemented code. For a Smart System this pre-programmed and therefore fixed operating code has to be developed in the direction of a processes similar to human cognitive behaviour. This might open the chance for advanced decision making in complex situations (e.g. in complexe traffic situations).

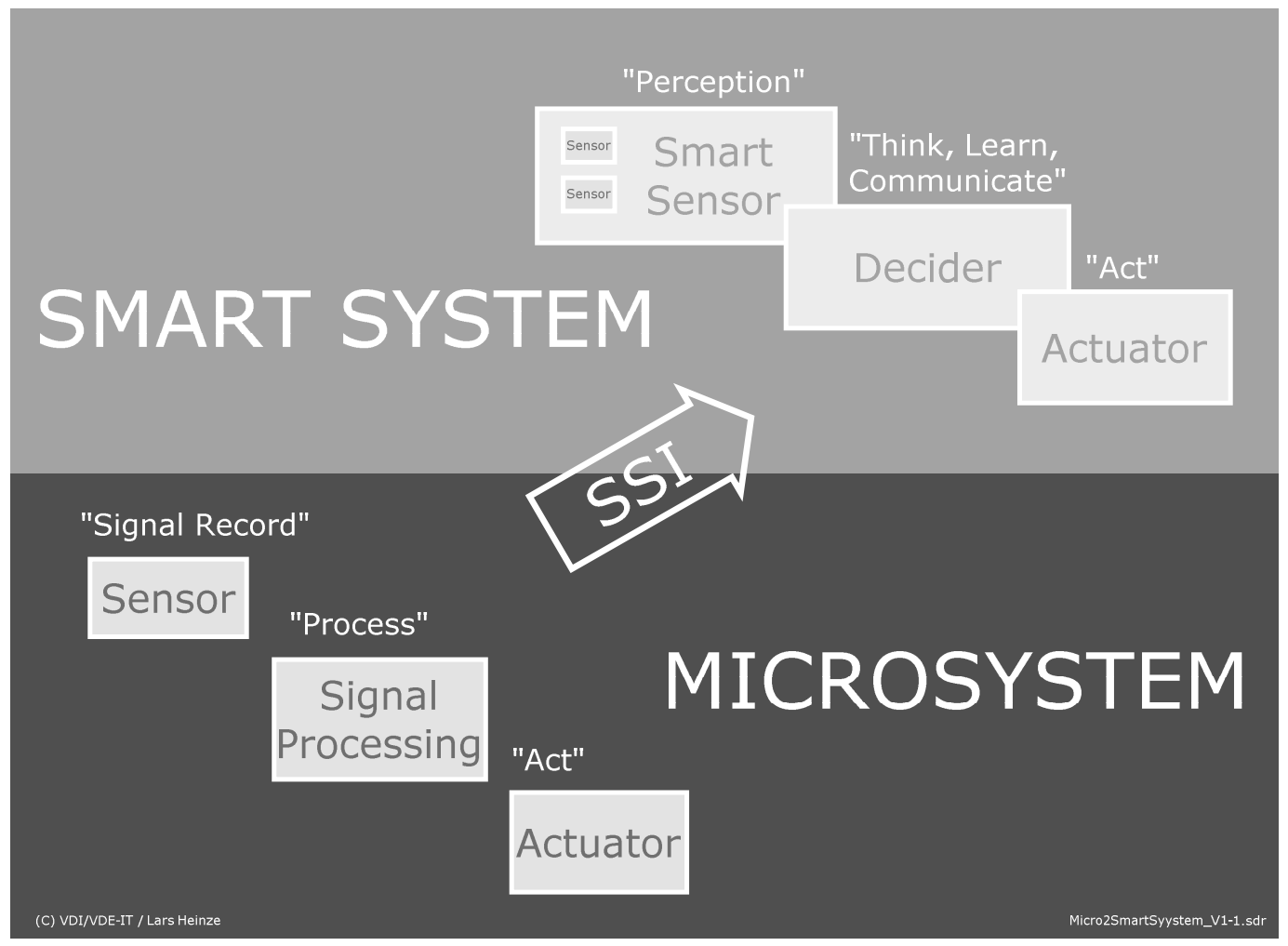

Figure 1: Transition from Microsystems to Smart Systems using Cognitive Sciences in the Frame of SSI 


\section{Requirements for Smart Sensors}

Cognitve processes are based on the perception of the evironment. Therefore a Smart System will need Smart Sensor(s). This leads to some main requirements:

- System Integration for highly miniaturised sensor(-element)s in order to be able to place more sensors - Integration of elements with cognitve abilities

- Self-sufficient energy base for the autonomy of smart sensors to be part of the environment

Cognitve elements will enable smart sensing as expressed by higher developed beings like humans. the cognitive abilities needed might involve e.g.

- $\quad$ universal mathematical models for the description of cognitive processes (e.g. for learning processes in the brain)

- how attention and improvisation emerge from perception

- multi-modal cognition (e.g. combination of listening and seeing)

- perception and analysis of complex situations based on simple basic rules and algorithms

Further requirements for Smart Sensors might arise regarding System Integration technologies for the miniaturisation of Smart Sensors e.g. to enable flexible geometry to adopt sensors to different environmaental situations

The new quality of such Smart Sensors would be:

- transmission of more or less complex information instead of a pure sensor signal

- possibilities to adapt the sensing to changing situations in the environment and the possibilies of the actuator part ("adaptivity" e.g. if enviromental conditions change into a range not foreseen in the beginning) 